\title{
Contrast-enhanced endoscopic ultrasonography (CE-EUS) findings in adrenal metastasis from renal cell carcinoma
}

\author{
Susumu Hijioka • Akira Sawaki • Nobumasa Mizuno - Kazuo Hara • \\ Mohamed A. Mekky • Hussein El-Amin - Zain El-Abdeen Ahmed Sayed • \\ Mssahiro Tajika $\cdot$ Yasumasa Niwa $\cdot$ Kenji Yamao
}

Received: 8 September 2010/Accepted: 26 November 2010/Published online: 5 January 2011

(C) The Japan Society of Ultrasonics in Medicine 2011

\begin{abstract}
Solitary adrenal metastasis is often difficult to distinguish from benign adrenal tumor using only plain computed tomography (CT) scanning. We describe a solitary left adrenal gland mass in a patient who had undergone simultaneous gastrectomy and right nephrectomy for advanced gastric cancer and renal cell carcinoma (RCC), respectively. Contrast-enhanced endoscopic ultrasonography (CE-EUS) findings indicated a hypervascular adrenal mass, and EUS-guided fine needle aspiration (EUS-FNA) revealed clear cell carcinoma. Adrenalectomy confirmed metastatic clear cell carcinoma of the kidney.
\end{abstract}

Keywords Cancer $\cdot$ Diagnostic imaging $\cdot$ Contrast agent $\cdot$ Kidney

$\begin{array}{ll}\text { Abbreviations } \\ \text { CT } & \text { Computed tomography } \\ \text { EUS } & \text { Endoscopic ultrasonography } \\ \text { MRI } & \text { Magnetic resonance imaging } \\ \text { FNA } & \text { Fine needle aspiration }\end{array}$

S. Hijioka $(\bowtie) \cdot$ A. Sawaki $\cdot$ N. Mizuno $\cdot$ K. Hara $\cdot$ K. Yamao Department of Gastroenterology, Aichi Cancer Center Hospital, 1-1 Kanokoden, Chikusa-ku, Nagoya, Aichi 464-8681, Japan e-mail: rizasusu@aichi-cc.jp

M. A. Mekky

Department of Tropical Medicine and Gastroenterology, Assiut University Hospital, Assiut, Egypt

H. El-Amin · Z. El-Abdeen Ahmed Sayed

Department of Internal Medicine and Gastroenterology, Assiut University Hospital, Assiut, Egypt

M. Tajika $\cdot$ Y. Niwa

Department of Endoscopy, Aichi Cancer Center Hospital, Nagoya, Japan $\begin{array}{ll}\text { RCC } & \text { Renal cell carcinoma } \\ \text { CE-EUS } & \text { Contrast-enhanced endoscopic ultrasonography }\end{array}$

\section{Introduction}

Renal cell carcinoma (RCC) is highly metastatic. Isolated solitary adrenal metastasis from RCC is relatively uncommon and occurs at a rate of about $10 \%$ [1, 2]. Synchronous RCC metastasis to the contralateral adrenal gland is very rare, clinically presenting in only $0.51 \%$ of patients $[1,2]$. On the other hand, adrenal adenomas are often detected incidentally during screening workups or follow-ups for other co-existing diseases. It is clinically important to differentiate between adenoma and metastatic adrenal tumors, especially in those with cancer or a history of treated cancer. Although some diagnostic imaging procedures have been established to distinguish these lesions, the implementation of contrastenhanced endoscopic ultrasonography (CE-EUS) to investigate adrenal masses has not yet been reported. Here, we describe a left adrenal mass that was diagnosed by CE-EUS as a hypervascular lesion, in which subsequent EUS-guided fine needle aspiration (EUS-FNA) revealed a metastasized contralateral renal cell carcinoma.

\section{Case presentation}

A 60-year-old man had undergone a simultaneous gastrectomy and right nephrectomy for advanced gastric cancer and $\mathrm{RCC}$, respectively. The final pathological diagnosis of the gastric cancer was Stage 3 (pT4, pN1, sP0, sH0, sM0) moderately differentiated tubular adenocarcinoma 
according to the Japanese classification of gastric carcinoma. On the other hand, the final diagnosis of the RCC was Stage 3 [pT3b G1>G2 INF $\alpha \mathrm{V}(+)$ ] clear cell carcinoma according to the Japanese classification of RCC. A routine follow-up plain computed tomography (CT) scan 2 years later detected a mass on the left adrenal gland (Fig. 1). A diagnostic workup comprising a panel of laboratory and imaging studies was planned. The laboratory findings were within normal ranges except for impaired renal chemistry (the patient had a high creatinine level, $2.1 \mathrm{mg} / \mathrm{ml}$ ). An EUS examination was performed using an electronic radial scanning echoendoscope (GF-UM 200, Olympus, Tokyo, Japan) connected to a processor (Aloka ProSound alpha-10). The left adrenal gland can be identified and imaged from the fundus of the stomach. The right adrenal gland, particularly when enlarged, can be examined from the second portion of the duodenum. Stepwise identification of the left and right adrenal glands via the gastric and duodenal approaches, respectively, has been standardized [3].

Our patient had a $25-\mathrm{mm}$ hypoechoic mass with a regular outline and a relatively heterogeneous internal echo but with preserved parenchyma (Fig. 2). Contrast-enhanced CT and magnetic resonance imaging (MRI) were not options, due to renal impairment; hence, EUS with contrast using Sonazoid $^{\circledR}$ (Daiichi-Sankyo, Tokyo, Japan) was planned to resolve the burden of renal dysfunction and to further examine the vasculature of the mass. After reducing the mechanical index to 0.15 , an intravenous injection of Sonazoid ${ }^{\circledR}$ resulted in visualization of a hypervascular mass in the early vascular phase and a delayed contrast effect in the post-vascular phase. A network of flow signals was evident within $18 \mathrm{~s}$ of CE-EUS (Fig. 3a), followed by a

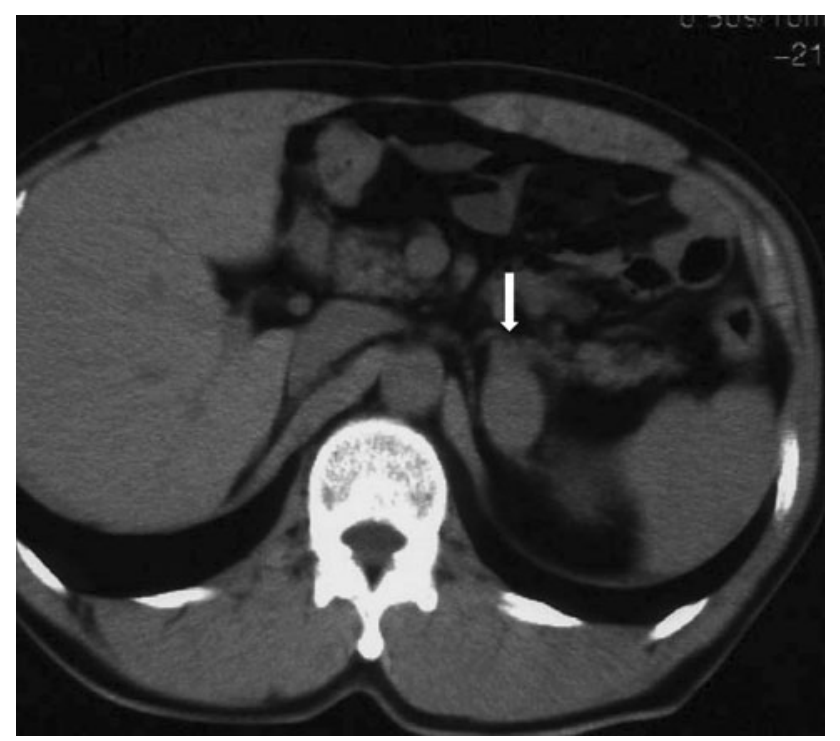

Fig. 1 Plain CT scan image shows a mass (arrow) on the left adrenal gland hypervascular signal at $25 \mathrm{~s}$ (Fig. 3b). The flow signals inside the tumor were amplified for $120 \mathrm{~s}$ (Fig. 3c).

Tissues were sampled using EUS-FNA with a convex array echoendoscope (GF-UCT240; Olympus, Tokyo, Japan) and a 22-gauge needle (NA-200H-8022; Olympus, Tokyo, Japan) (Fig. 4). Histopathologically, an H\&Estained sample revealed atypical acidophilic clear cells (Fig. 5a). Immunohistochemical staining revealed that the tissues were reactive to CD10 antibody (Fig. 5b), which was consistent with a diagnosis of clear cell carcinoma and indicated contralateral adrenal metastasis from the original RCC. Left adrenalectomy was performed, and the final pathological diagnosis was metastatic clear cell carcinoma of the kidney (Fig. 6a, b).

\section{Discussion}

It is critical to differentiate between adenomas and metastatic adrenal tumors in patients with cancer or a history of treated cancer. Lipid content and enhancement characteristics are two main imaging features of adrenal lesions that are useful in differentiating adenomas from metastases. Most adenomas (about 80\%) contain lipid that causes low attenuation on CT scans [4] and thus unenhanced CT scans can reliably characterize adrenal adenomas. However, a precise cut-off threshold between benign and malignant lesions has not been established [5, 6]. Chemical shift MRI can be used to characterize lipid-rich adenomas with an accuracy similar to that of non-enhanced CT scans [7], but adenomas with small amounts of lipid will not be accurately discriminated by either imaging modality [8].

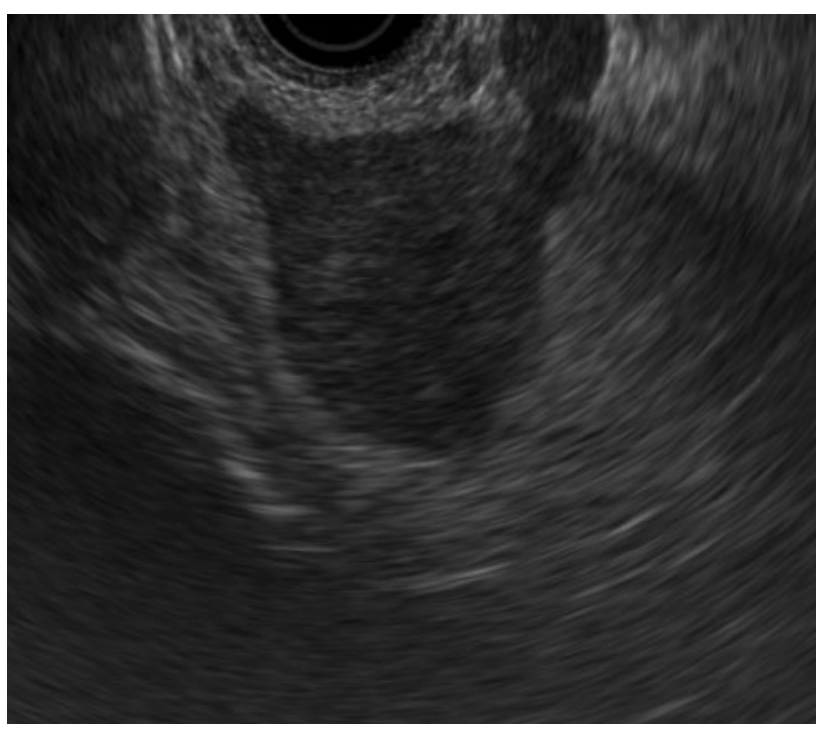

Fig. 2 Convex EUS image of the left adrenal grand shows a wellcircumscribed hypoechoic mass and relatively heterogeneous internal echo 
Fig. 3 CE-EUS with

Sonazoid ${ }^{\circledR}$ shows a network of flow signals at $18 \mathrm{~s}$ (a) and hypervascularity at $25 \mathrm{~s}(\mathbf{b})$. Flow signals inside the tumor were amplified up to $120 \mathrm{~s}$ (c)
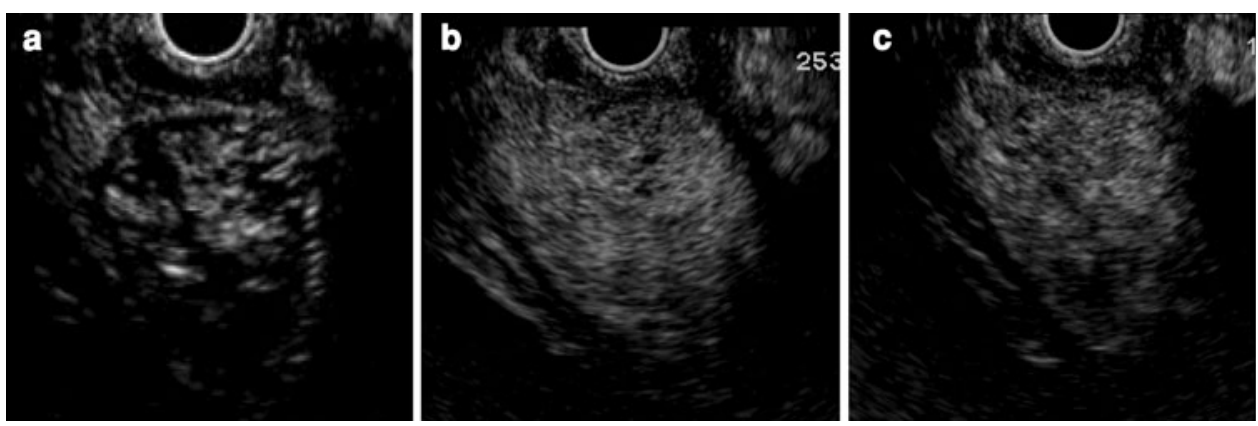

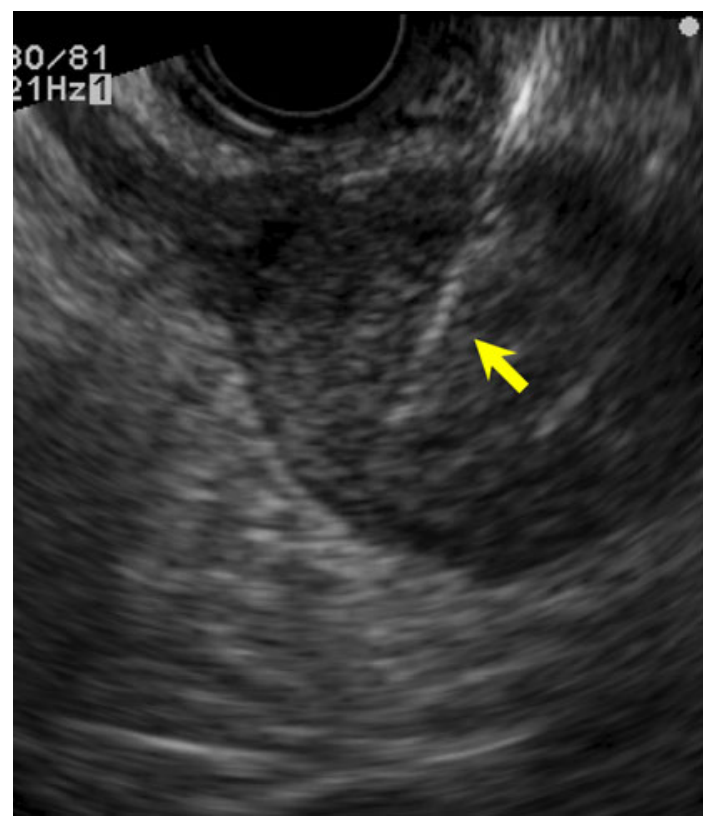

Fig. 4 EUS-FNA with needle inserted into the mass (arrow)

For the remaining $20 \%$ of lipid-poor adenomas with similar attenuation as metastases on unenhanced CT scans, CT enhancement washout calculations might differentiate adenomas from metastases, as adenomas are mildly enhanced and washout is rapid [4]. Shinozaki et al. [9] found that obvious vascularity of an adrenal mass favors a diagnosis of metastasis rather than adenoma, and thus contrast imaging material is required to define this vascular profile. Since renal impairment can hamper contrast in both CT and MRI, another contrast-based imaging modality, such as CE ultrasound, should be considered. Anatomical obstacles that hide the adrenal gland make it difficult to detect these lesions with conventional ultrasound and even with CE ultrasound. The advent of EUS using a convex array echoendoscope was a major breakthrough in visualizing such remote lesions, rendering them amenable to both imaging and sampling [10]. The limited band and low acoustic power of EUS renders contrast harmonic imaging impossible, but the development of novel US contrast agents [11] that can produce harmonic signals at low acoustic power [12] has recently removed this disadvantage [13]. Sonazoid ${ }^{\circledR}$ is a second-generation US contrast agent that can differentiate intra-tumor flow signals with characteristics similar to those of transabdominal US [14]. Moreover, vascular CE-EUS images are more precise than CT and MR images [14], with an additional advantage of being a microbubble contrast that is eliminated by exhalation [15] and hence independent of renal perfusion capacity, which favors its use in this case in particular [16]. The adrenal gland normally appears on EUS as a seagull-shaped organ without nodularity. In the present case, obvious hypervascularity was detected by CE-EUS in a small lesion in the vascular phase when compared with surrounding tissue. Amplification for $90 \mathrm{~s}$ showed some avascular areas that suggested a necrotic compartment. These findings indicated RCC metastasis rather than adenoma or gastric carcinoma metastasis. To the best of our knowledge, adrenal metastasis from RCC has never been identified using CE-EUS with Sonazoid ${ }^{\circledR}$. Our suspected diagnosis was augmented by EUS-FNA findings.

The safety and utility of EUS-FNA for adrenal lesions has been supported by several investigators [3, 10, 17], who reported safe and accurate sampling from the adrenal gland with very favorable profiles compared with percutaneous approaches. This might be because the only organ traversed by the needle is the gastric wall. Meanwhile, cautious evaluation of the needle track is usually performed using a prior Doppler check to avoid puncturing any intervening vessels. Adequate suction of the gastric contents during the procedure is also a valuable step in reducing spoilage of the aspirate with gastric juice. Information obtained by EUS-FNA influenced the management of our patient and facilitated decision making. We therefore recommend using EUS-FNA in the diagnostic workup of patients with suspected adrenal lesions.

\section{Conclusion}

We describe a patient with a relatively rare contralateral adrenal metastasis of renal cell carcinoma. We contend that CE-EUS with Sonazoid ${ }^{\circledR}$ could become the modality of 
Fig. 5 Typical acidophilic clear cells stained with $\mathrm{H} \& \mathrm{E}, \times 400$ (a) and positive immunohistochemical staining for CD10, $\times 400$ (b)
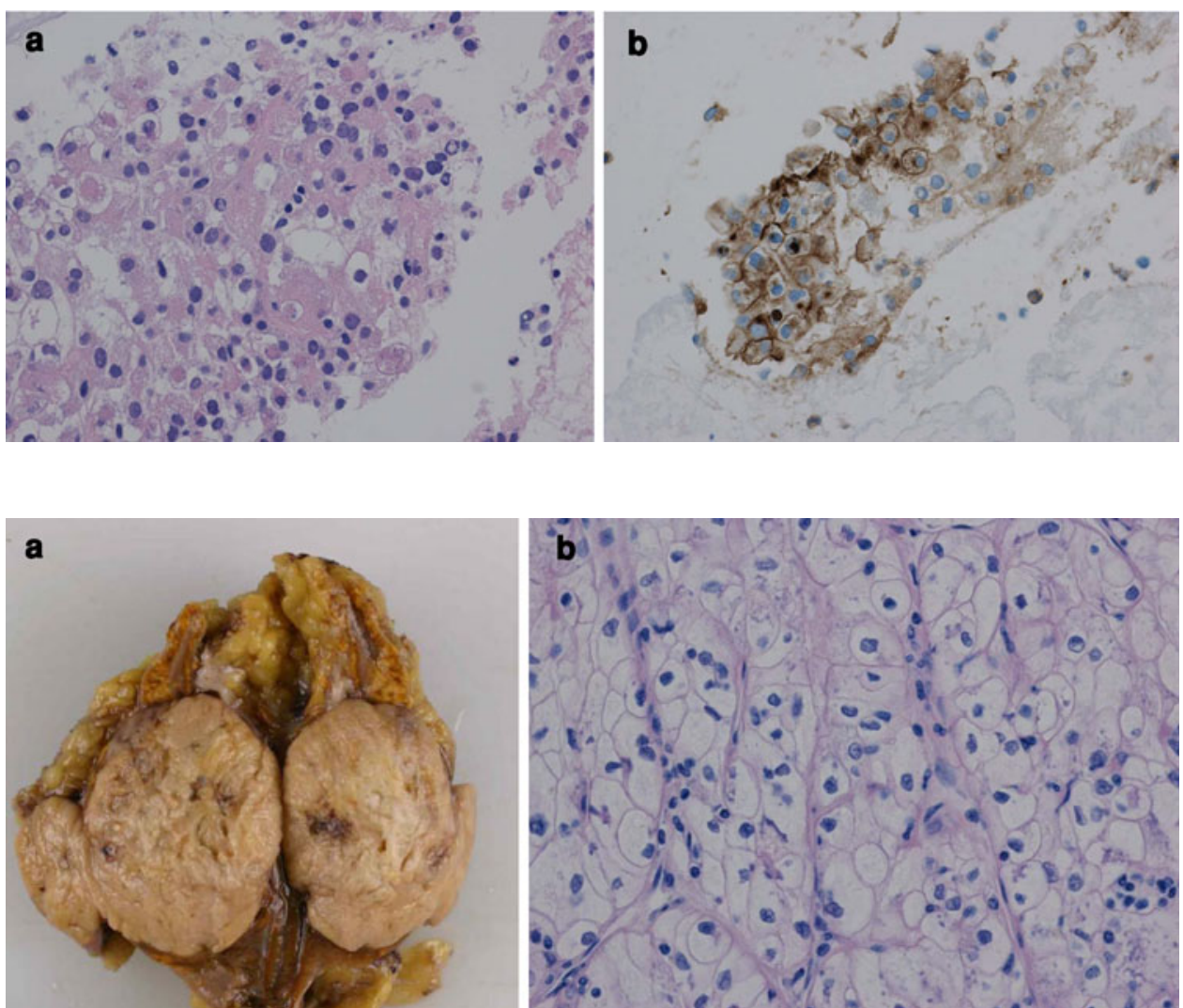

Fig. 6 Well-demarcated resected specimen (a) is histologically composed of typical clear cells with an alveolar structure (b) choice to distinguish between adrenal metastasis and adrenal adenoma, and subsequent EUS-FNA might be a useful tool for reaching a final diagnosis.

\section{References}

1. Lau WK, Zincke H, Lohse CM, et al. Contralateral adrenal metastasis of renal cell carcinoma: treatment, outcome and a review. BJU Int. 2003;91:775-9.

2. Kuczyk M, Wegener G, Jonas U. The therapeutic value of adrenalectomy in case of solitary metastatic spread originating from primary renal cell cancer. Eur Urol. 2005;48:252-7.

3. Eloubeidi MA, Black KR, Tamhane A, et al. A large single-center experience of EUS-guided FNA of the left and right adrenal glands: diagnostic utility and impact on patient management. Gastrointest Endosc. 2010;71:745-53.

4. Peña C, Boland $G$, Hahn P, et al. Characterization of indeterminate (lipid-poor) adrenal masses: use of washout characteristics at contrast-enhanced CT. Radiology. 2000;217:798.

5. Boland G, Hahn P, Peña C, et al. Adrenal masses: characterization with delayed contrast-enhanced CT. Radiology. 1997;202:693.

6. Miyake H, Takaki H, Matsumoto S, et al. Adrenal nonhyperfunctioning adenoma and nonadenoma: $\mathrm{CT}$ attenuation value as discriminative index. Abdom Imaging. 1995;20:559-62.

7. Heinz-Peer G, Honigschnabl S, Schneider B, et al. Characterization of adrenal masses using MR imaging with histopathologic correlation. Am J Roentgenol. 1999;173:15.

8. Korobkin M. CT characterization of adrenal masses: the time has come. Radiology. 2000;217:629.
9. Shinozaki K, Yoshimitsu K, Honda H, et al. Metastatic adrenal tumor from clear-cell renal cell carcinoma: a pitfall of chemical shift MR imaging. Abdom Imaging. 2001;26:439-42.

10. DeWitt J, Alsatie M, LeBlanc J, et al. Endoscopic ultrasoundguided fine-needle aspiration of left adrenal gland masses. Endoscopy. 2007;39:65-71.

11. Kitano M, Sakamoto H, Matsui U, et al. A novel perfusion imaging technique of the pancreas: contrast-enhanced harmonic EUS (with video). Gastrointest Endosc. 2008;67:141-50.

12. Rickes S, Uhle C, Kahl S, et al. Echo enhanced ultrasound: a new valid initial imaging approach for severe acute pancreatitis. Gut. 2006;55:74-8.

13. Kitano M. Omentoplasty in thoracic surgery. Gen Thorac Cardiovasc Surg. 2008;56:483-9.

14. Imazu H, Uchiyama Y, Matsunaga K, et al. Contrast-enhanced harmonic EUS with novel ultrasonographic contrast (Sonazoid) in the preoperative $\mathrm{T}$-staging for pancreaticobiliary malignancies. Scand J Gastroenterol. 2010;45:732-8.

15. Uran S, Landmark K, Normann PT, et al. A respiration-metabolism chamber system and a GC-MS method developed for studying exhalation of perfluorobutane in rats after intravenous injection of the ultrasound contrast agent Sonazoid (TM). J Pharm Biomed Anal. 2005;39:746-51.

16. Tsuruoka K, Yasuda T, Koitabashi K, et al. Evaluation of renal microcirculation by contrast-enhanced ultrasound with sonazoid (TM) as a contrast agent. Int Heart J. 2010;51:176-82.

17. DeWitt J, LeBlanc J, McHenry L, et al. Endoscopic ultrasoundguided fine needle aspiration cytology of solid liver lesions: a large single-center experience. Am J Gastroenterol. 2003;98: 1976-81. 